\title{
COPING WITH ANXIETY, DEPRESSION, BURDEN AND QUALITY OF LIFE IN INFORMAL PRIMARY CAREGIVERS OF COMMUNITY- DWELLING INDIVIDUALS WITH DEMENTIA
}

\author{
M. Muscat ${ }^{1}$, C. Scerri ${ }^{2}$
}

\begin{abstract}
Objective: This study aimed to investigate how informal primary caregivers of individuals with dementia living in the community cope with caring-related measures of anxiety, depression, burden and quality of life. Participants and Design: Participants included 60 informal caregivers (23 males and 37 females) of community-dwelling individuals with dementia who attended a state-run geriatric day hospital in Malta. Caregiver measures included the Hospital Anxiety and Depression Scale, the World Health Organization Quality of Life-BREF and Brief COPE questionnaires, and Zarit Burden Interview. The Mini Mental State Examination and Barthel Index of Activities of Daily Living scores were used to determine the degree of cognitive impairment and performance in activities of daily living of care-recipients. Results: Informal caregivers experienced anxiety and depression with both emotional distress states negatively affecting all quality of life domains. Depression and burden experienced by informal primary caregivers showed a strong association with individuals with dementia-related variables such as age, cognitive impairment and activities of daily living scores. The use of dysfunctional coping strategies was found to be related to caregivers' emotional distress, low quality of life and burden. Conclusion: The findings indicate that informal primary caregivers experienced anxiety and depression, had a lower quality of life, and feel burdened during their caring role. However, caregivers making use of emotion-focused coping strategies were found to be protected against emotional distress. The results highlight the need of providing support services aimed at promoting the psychological wellbeing of informal carers of community-dwelling individuals with dementia.
\end{abstract}

Key words: Caregiver, coping, dementia, emotional distress, quality of life.

\section{Introduction}

Dementia is a clinical term referring to a group of brain disorders characterized by progressive deterioration of cognitive abilities. In 2015, it was estimated that $1.5 \%$ of the population in Malta had dementia, a figure that is projected to more than double in the next 30 years (1). This, in conjunction with a demographic shift favouring a progressive increase in the elderly population, will add to a growing burden on family members who, in the majority of cases, provide informal care for these individuals at home (2).

At present there is no cure for the most common forms of dementia and consequently the main focus lies in promoting the wellbeing and providing optimal quality of life for the individual with dementia and their caregivers (3). Previous research has demonstrated that high levels of neuropsychiatric symptoms in dementia

1. Department of Gerontology, Faculty for Social Wellbeing; 2. Department of Pathology, Faculty of Medicine and Surgery, University of Malta, Msida, Malta MSD 2080

Corresponding Author: Charles Scerri PhD, Department of Pathology, Faculty of Medicine and Surgery, University of Malta, Msida MSD 2080, Malta; Tel.: +356 23402905; Fax: +356 21320281; E-mail: charles.scerri@um.edu.mt caregivers leads to a reduction in their quality of life (4) and caregiver burden is mostly related to the caregiver ability to cope with the situation (5). A number of coping strategies and styles adopted by dementia caregivers have been proposed with those based on emotional support and problem-focused strategies being associated with caregiver wellbeing and positive outcomes (6-8).

The main objective of this study was to investigate how coping strategies and styles used by informal primary caregivers caring for an individual with dementia living in the community influence their levels of anxiety, depression, burden and quality of life. This category of caregivers was selected as previous research have shown that, in Malta, rather than resorting to institutionalization of the person with dementia, a unique model of care based on reliance on families through a rotation pattern of care by different family members is preferred (2). Therefore, the identification of predictive factors that enhance the wellbeing of caregivers of individuals with dementia living in the community would not only ensure that health and social support programmes are designed to meet the desired needs but would further delay institutionalization of the carerecipient (9). 


\section{Methods}

The study was conducted in Malta from early January to the end of May 2015. Participants were informal primary caregivers of individuals with dementia attending a state-run geriatric day hospital who had received a formal diagnosis of dementia by a medical specialist. A convenience sample of caregivers $(\mathrm{n}=74)$ was selected with selection criteria being attendance to the day hospital by the individuals with dementia, individuals with dementia living in the community, caregiver residing in the same or separate household and being the primary care provider. Participants were contacted by telephone following which 60 agreed to take part in the study. Sociodemographic data of caregivers included age, gender, occupation, relationship with individuals with dementia, marital status, level of education, whether they were living with the individuals with dementia, duration of caregiving in years, number of contact hours per day and whether they were formally diagnosed with anxiety/depression during their caregiving role. Information on individuals with dementia included age, gender and dementia type. The levels of cognitive impairment and activities of daily living of individuals with dementia were based on the Mini Mental State Examination (MMSE) (10) and Barthel Index of Activities of Daily Living (BI-ADL) (11) scores measured by a geriatric medical specialist prior to study commencement.

The research instruments used in assessing caregivers included the Hospital Anxiety and Depression Scale (HADS), the World Health Organization Quality of LifeBREF (WHOQOL-BREF) and Brief COPE questionnaires, and Zarit Burden Interview (ZBI). The HADS consists of two seven-item subscales, one related to anxiety and the other to depression (12). Each item carries a four-point scale (0-3) with the total possible score for both anxiety and depression ranging from $0-21$. Scores are categorized as normal (0-7), mild (8-10), moderate (11-14) and severe (15-21). The WHOQOL-BREF comprises 26 items grouped under domains for physical health, psychological health, social relationships and environmental health (13). Each item is rated on a 5-point Likert scale with higher scores denoting better quality of life. The Zarit Burden Interview consists of 22 items with a 5-point Likert scale ranging from 0 (never) to 4 (nearly always) (14) whereas the Brief COPE measures a number of different coping strategies and consists of 14 subscales with two questions per style with a 4-point Likert scale ranging from 1 (I haven't been doing this at all) to 4 (I've been doing this a lot) (15). The coping strategies are divided into 3 domains: emotion-focus (use of emotional support, positive reframing, acceptance, religion, humour), problem-focus (active coping, planning, use of instrumental support) and dysfunctional coping (venting, denial, substance use, behavioral disengagement, self-distraction, self-blame) (16).
All interviews with informal primary caregivers were carried out face-to-face in English language at the participants' residence. The study was approved by the Faculty of Social Wellbeing Ethics Committee and the Research Ethics Committee of the University of Malta. Permission was also granted by the management of the day hospital. Participants were guaranteed confidentiality and anonymity and were free to withdraw at any stage of the interview without giving a reason. Written consent was obtained from all participants. Depending on cognitive impairment, consent from individuals with dementia was obtained either directly or by proxy.

Descriptive statistics were used to summarize sociodemographic and clinical data of participants as percentages, means and standard deviation (SD). Relationships between anxiety, depression, quality of life, burden and coping scores with sociodemographic characteristics and clinical data were analysed using Student's t-test for two groups of data and one factor ANOVA with post hoc comparisons using Tukey for multiple groups. In the event that the data were not normally distributed as determined by the Shapiro-Wilk test, the Mann-Whitney U test was applied.

To study the degree to which anxiety and depression, quality of life and burden experienced by caregivers was related to their coping strategies and styles, the Pearson's correlation coefficient ( $\mathrm{r}$ ) analysis was conducted. All research instruments used for caregivers' measures were assessed for internal consistency using the Cronbach's alpha test. Data analysis was conducted using PASW Statistics (version 20.0) with significance level set at 0.05.

\section{Results}

\section{Characteristics of participants}

Descriptive characteristics of individuals with dementia and their informal primary caregivers are presented in Table 1. Care-recipients had a mean age of 77.5 years (range: 46 - 92) and showed moderate functional dependency levels. With respect to the severity of cognitive function, $36.7 \%$ had mild, $25.0 \%$ moderate and $38.3 \%$ severe cognitive impairment. A significant positive correlation was observed between MMSE and BI-ADL $(r=0.801, \mathrm{p}<0.001)$ indicating that individuals with dementia having severe cognitive impairment were the most functionally dependent.

Informal primary caregivers were mostly females, married, had a secondary level of education, unemployed and living in the same household. The duration of caregiving role varied from 1 to 25 years with an average of 4.5 years whereas the average number of contact hours spent in daily caregiving was 16.2 hours. A third of participants indicated that they spend 24 hours per day in their caregiving role. 


\section{Table 1}

Sociodemographic characteristics and clinical data of individuals with dementia and their informal primary caregivers

(AD, Alzheimer's disease; BI-ADL, Barthel Index of Activities of Daily Living; ipCG, informal primary caregiver; IWD, individual with dementia; LBD, Lewy-body dementia;

MMSE, Mini-Mental State Examination; VaD, vascular dementia)

\begin{tabular}{|c|c|c|c|c|}
\hline \multirow[b]{2}{*}{ Variables } & \multicolumn{2}{|c|}{ IWD $(N=60)$} & \multicolumn{2}{|c|}{ ipCG $(N=60)$} \\
\hline & n $(\%)$ & Mean \pm SD & n $(\%)$ & Mean $\pm \mathrm{SD}$ \\
\hline \multicolumn{5}{|l|}{ Gender } \\
\hline Male & $25(41.7)$ & & $23(38.3)$ & \\
\hline Female & $35(58.3)$ & & $37(61.7)$ & \\
\hline Age (years) & & $77.5 \pm 8.9$ & & $63.8 \pm 10.8$ \\
\hline MMSE score & & $14.6 \pm 9.2$ & & \\
\hline BI-ADL score & & $13.0 \pm 6.8$ & & \\
\hline \multicolumn{5}{|l|}{ Dementia type } \\
\hline $\mathrm{AD}$ & $51(85.0)$ & & & \\
\hline $\mathrm{VaD}$ & $6(10.0)$ & & & \\
\hline LBD & $3(5.0)$ & & & \\
\hline \multicolumn{5}{|l|}{ Status } \\
\hline Single & & & $8(13.3)$ & \\
\hline Married & & & $50(83.3)$ & \\
\hline Widowed & & & $2(3.4)$ & \\
\hline \multicolumn{5}{|l|}{ Education } \\
\hline Primary & & & $19(31.7)$ & \\
\hline Secondary & & & $30(50.0)$ & \\
\hline Post-secondary & & & $11(18.3)$ & \\
\hline \multicolumn{5}{|l|}{ Occupation } \\
\hline Employed & & & $20(33.3)$ & \\
\hline Unemployed & & & $20(33.3)$ & \\
\hline \multicolumn{5}{|l|}{ Relationship with IWD } \\
\hline Spouse & & & $29(48.3)$ & \\
\hline Son & & & $8(13.3)$ & \\
\hline Daughter & & & $17(28.3)$ & \\
\hline Sibling & & & $4(6.7)$ & \\
\hline Friend & & & $2(3.3)$ & \\
\hline \multicolumn{5}{|l|}{ Caregiving } \\
\hline Number of years & & & & $4.5 \pm 4.1$ \\
\hline Number of hours/day & & & & $16.2 \pm 9.5$ \\
\hline \multicolumn{5}{|l|}{ Living with IWD } \\
\hline Yes & & & $40(66.7)$ & \\
\hline No & & & $20(33.3)$ & \\
\hline \multicolumn{5}{|c|}{ Diagnosis of anxiety/depression during caregiving } \\
\hline Yes & & & $19(31.7)$ & \\
\hline No & & & $41(68.3)$ & \\
\hline
\end{tabular}

\section{Informal primary caregivers' anxiety and depression}

The mean anxiety score for caregivers was found to be significantly higher than the mean depression score $(F$ $=8.594, \mathrm{p}<0.001)$ denoting that caregivers experienced more anxiety than depression during their caring role (Table 2). Furthermore, anxiety and depression were found to be significantly correlated $(\mathrm{r}=0.777, \mathrm{p}<0.001)$ indicating that caregivers with high anxiety scores tended to have higher levels of depression. Caregivers who had a lower level of education, cared for individuals with dementia with low cognitive and functional scores, and formally diagnosed with anxiety/depression during the caregiving role experienced higher levels of anxiety and depression.

\section{Informal primary caregivers' quality of life}

As indicated in Table 2, caregivers who were employed, had a post-secondary level of education and have not been formally diagnosed with anxiety/ depression during their caregiving role had higher quality of life scores. Amongst the domains tested, the two highest average standardised scores were reported for the environment and psychological domains with social relationships scoring the lowest. The social relationships domain was found to be significantly correlated with the age of the individual with dementia $(\mathrm{r}=0.264, \mathrm{p}=0.042)$ and caregiver $(\mathrm{r}=-0.277, \mathrm{p}=0.032)$ and related to whether the caregiver was living in the same household, relationship with the individual with dementia and the educational status of the caregiver. The latter was also found to similarly affect the environment domain.

\section{Informal primary caregivers' burden}

Approximately half of the caregivers participating in this study $(46.7 \%, \mathrm{n}=28)$ indicated that they experienced moderate to severe/severe burden whilst caring for an individual with dementia. A significant inverse correlation was found between the age of the individual with dementia and the burden score $(\mathrm{r}=-0.299, \mathrm{p}=$ 0.020) suggesting that the younger the individual with dementia, the greater the burden on the caregiver. A low BI-ADL score and a formal diagnosis of anxiety/ depression in the caregiver were also found to be significantly related to higher caregiver burden (Table 2).

\section{Informal primary caregivers' coping strategies}

The mean rating scores for emotion-focus and problemfocus strategies were similar and significantly higher than dysfunctional coping $(p<0.001)$ denoting that the latter was the coping strategy that was used the least by the caregivers (Table 3). Moreover, dysfunctional coping was inversely correlated with the age of the care-recipient 


\section{Table 2}

Relationship between anxiety, depression, quality of life and burden scores with sociodemographic characteristics and clinical data of individuals with dementia and their informal primary caregivers $(\alpha$, Cronbach's alpha; BI-ADL,

Barthel Index of Activities of Daily Living; ipCG, informal primary caregiver; IWD, individual with dementia; MMSE, Mini-Mental State Examination)

\begin{tabular}{|c|c|c|c|}
\hline & $\begin{array}{c}\text { Mean scores } \\
\pm \text { SD }\end{array}$ & $\%$ & P-value (IWD, ipCG characteristics) \\
\hline \multicolumn{4}{|l|}{$\operatorname{HADS}(\alpha=0.895)$} \\
\hline Anxiety (7 items) & $11.35 \pm 5.98$ & & 0.018 (level of education-ipCG) \\
\hline Normal (0-7) & & 35.0 & \\
\hline Mild (8-10) & & 11.7 & \\
\hline Moderate (11-14) & & 20.0 & \\
\hline Severe (15-21) & & 33.3 & \\
\hline \multirow[t]{3}{*}{ Depression (7 items) } & $6.87 \pm 5.23$ & & 0.025 (MMSE score-IWD) \\
\hline & & & 0.019 (BI-ADL score-IWD) \\
\hline & & & 0.005 (anxiety / depression-ipCG) \\
\hline Normal (0-7) & & 66.7 & \\
\hline Mild (8-10) & & 8.3 & \\
\hline Moderate (11-14) & & 15.0 & \\
\hline Severe $(15-21)$ & & 10.0 & \\
\hline \multicolumn{4}{|l|}{ WHOQOL-BREF ( $\alpha=0.915)$} \\
\hline \multirow[t]{3}{*}{ Total } & $86.65 \pm 15.40$ & & 0.038 (occupation-ipCG) \\
\hline & & & $<0.001$ (level of education-ipCG) \\
\hline & & & 0.045 (anxiety / depression-ipCG) \\
\hline Physical health (7 items) & $53.57 \pm 11.93$ & & 0.017 (gender-ipCG) \\
\hline Psychological (6 items) & $58.62 \pm 12.08$ & & \\
\hline \multirow[t]{4}{*}{ Social relationships (3 items) } & $38.27 \pm 25.67$ & & 0.003 (age-ipCG) \\
\hline & & & < 0.001 (relationship with IWD) \\
\hline & & & $<0.001$ (level of education-ipCG) \\
\hline & & & 0.001 (living with IWD) \\
\hline Environment (8 items) & $61.65 \pm 15.39$ & & 0.001 (level of education-ipCG) \\
\hline \multicolumn{4}{|l|}{$\mathrm{ZBI}(\alpha=0.875)$} \\
\hline \multirow[t]{3}{*}{ Total } & $39.33 \pm 17.33$ & & 0.040 (age-IWD) \\
\hline & & & 0.046 (BI-ADL score-IWD) \\
\hline & & & 0.001 (anxiety/depression-ipCG) \\
\hline Little or no burden $(<21)$ & & 15.0 & \\
\hline Mild to moderate (21-40) & & 38.3 & \\
\hline Moderate to severe (41-60) & & 31.7 & \\
\hline Severe $(>60)$ & & 15.0 & \\
\hline
\end{tabular}

( $r=-0.268, p=0.038)$ and significantly related to the increasing number of hours/day spent in caring and the presence of a formal diagnosis of anxiety/depression in the caregiver. Out of the 14 coping styles, the most frequently used were acceptance and active coping with substance abuse scoring the lowest. 
Table 3

Relationship between coping strategies and styles with sociodemographic characteristics and clinical data of individuals with dementia and their informal primary caregivers. Cronbach's $\alpha$ for the Brief COPE $=0.713$ (BI-ADL,

Barthel Index of Activities of Daily Living; ipCG, informal primary caregiver; IWD, individual with dementia)

Mean scores \pm SD

\section{P-value (IWD, ipCG characteristics)}

Coping strategies

Dysfunctional coping (12 items) $2.03 \pm 0.49$

0.018 (age-IWD)

0.005 (number of hours/day caring-ipCG)

0.001 (anxiety/depression-ipCG)

$\begin{array}{ll}\text { Problem-focus (6 items) } & 2.95 \pm 0.62 \\ \text { Emotion-focus (10 items) } & 2.96 \pm 0.66 \\ \text { Coping styles } & 7.00 \pm 1.54 \\ \text { Active coping } & \\ & \\ & \\ \text { Instrumental support } & 5.48 \pm 2.13 \\ \text { Planning } & 5.25 \pm 2.41 \\ \text { Acceptance } & 7.02 \pm 1.66 \\ \text { Emotional support } & 6.13 \pm 2.25 \\ \text { Humour } & 4.38 \pm 2.23 \\ \text { Positive reframing } & 5.92 \pm 2.18 \\ \text { Religion } & 6.10 \pm 2.74 \\ \text { Behavioural disengagement } & 4.27 \pm 2.19\end{array}$

0.012 (number of hours/day caring-ipCG)

0.037 (relationship with IWD)

0.018 (number of hours / day caring-ipCG)

0.019 (living with IWD)

0.018 (BI-ADL score-IWD)

0.045 (number of hours/day caring -ipCG)

0.018 (number of hours/day caring-ipCG)

0.035 (number of hours / day caring-ipCG)

0.043 (age-IWD)

0.040 (age-ipCG)

0.015 (anxiety / depression-ipCG)

Denial $\quad 4.45 \pm 2.16$

0.021 (age-IWD)

0.020 (status-ipCG)

0.023 (level of education-ipCG)

Self-distraction

Self-blame

Substance abuse
$4.05 \pm 1.66$

$4.00 \pm 1.74$

$2.73 \pm 1.54$
0.030 (gender-IWD)

0.036 (BI-ADL score-IWD)
Association between informal primary caregivers' measures of anxiety and depression, quality of life, burden and coping strategies and styles

In informal primary caregivers, higher levels of both anxiety and depression were found to be related to a reduction in the overall quality of life and increased burden (Table 4). Furthermore, caregivers who were using dysfunctional coping strategies experienced higher levels of anxiety and depression. The latter were also found to be positively correlated with the coping styles of planning, behavioral disengagement, denial and self-blame. Conversely, the use of acceptance, positive reframing and self-distraction were related to diminished emotional distress. Caregivers who reported high levels of burden had lower quality of life scores with all domains being affected except physical health. Burden scores were also found to be high in caregivers adopting dysfunctional coping strategies with relationships to coping styles similar to those reported for anxiety and depression. 
Table 4

Correlations between anxiety, depression, quality of life, burden and coping strategies and styles of informal primary caregivers of individuals with dementia (WHOQOL-BREF, World Health Organization Quality of LifeBREF; ZBI, Zarit Burden Interview). Only significant Pearson correlation coefficient (r) values reported $\left({ }^{*} \mathrm{P}<0.05\right.$, ** $\left.\mathrm{P}<0.01,{ }^{* * *} \mathrm{P}<0.001\right)$

\begin{tabular}{|c|c|c|c|c|c|c|c|c|c|}
\hline & & \multirow[b]{2}{*}{ Anxiety } & \multirow[b]{2}{*}{ Depression } & \multicolumn{6}{|c|}{ WHOQOL-BREF } \\
\hline & & & & $\begin{array}{c}\text { Physical } \\
\text { health }\end{array}$ & Psychological & $\begin{array}{c}\text { Social } \\
\text { relationships }\end{array}$ & Environment & Total & ZBI \\
\hline \multirow[t]{5}{*}{ WHOQOL-BREF } & Physical health & $-0.278^{*}$ & $-0.315^{*}$ & & & & & & \\
\hline & Psychological & $-0.399^{* *}$ & $-0.489^{* * *}$ & & & & & & \\
\hline & Social relationships & $-0.517^{* * *}$ & $-0.520^{* * *}$ & & & & & & \\
\hline & Environment & $-0.500^{* * *}$ & $-0.616^{* * *}$ & & & & & & \\
\hline & Total & $-0.547^{* * *}$ & $-0.665^{* * *}$ & & & & & & \\
\hline ZBI & & $0.744^{* * *}$ & $0.727^{\star \star *}$ & & $-0.468^{* * *}$ & $-0.450^{* * *}$ & $-0.583^{* * *}$ & $-0.625^{* * *}$ & \\
\hline \multirow[t]{3}{*}{ Coping strategies } & Dysfunctional coping & $0.450^{* * *}$ & $0.394^{* *}$ & & & & $-0.264^{*}$ & $-0.276^{*}$ & $0.422^{* * *}$ \\
\hline & Problem-focus & & & & & & & & \\
\hline & Emotion-focus & $-0.309^{*}$ & $-0.369^{* *}$ & & & & & & \\
\hline \multirow[t]{14}{*}{ Coping styles } & Active coping & & & & & & & & \\
\hline & Instrumental support & & & $0.286^{*}$ & & & & & \\
\hline & Planning & $0.284^{*}$ & & & $-0.380^{* *}$ & & $-0.320^{*}$ & $-0.285^{*}$ & $0.427^{* * *}$ \\
\hline & Acceptance & $-0.296^{*}$ & $-0.286^{*}$ & & & & & & \\
\hline & Emotional support & & & & & & & & \\
\hline & Humour & & & & & & & & \\
\hline & Positive reframing & $-0.418^{* * *}$ & $-0.440^{\star * *}$ & & $0.389^{* *}$ & $0.312^{*}$ & $0.340^{* *}$ & $0.387^{* *}$ & $-0.452^{* * *}$ \\
\hline & Religion & & & & & & & & \\
\hline & Behavioural disengagement & $0.576^{* * *}$ & $0.509^{* * *}$ & & $-0.315^{\star}$ & $-0.294^{*}$ & $-0.413^{* * *}$ & $-0.416^{* * *}$ & $0.583^{* * *}$ \\
\hline & Denial & $0.405^{* * *}$ & $0.282^{*}$ & & & & & & $-0.265^{*}$ \\
\hline & Self-distraction & $-0.354^{* *}$ & $-0.402^{* * *}$ & $0.331^{* *}$ & & $0.298^{*}$ & $0.334^{* *}$ & $0.350^{* *}$ & $-0.266^{*}$ \\
\hline & Self-blame & $0.527^{* * *}$ & $0.498^{* * *}$ & & & $-0.347^{* *}$ & $-0.376^{* *}$ & $-0.346^{* *}$ & $0.372^{\star *}$ \\
\hline & Substance abuse & & & & & & & & \\
\hline & Venting & & & & & & & & \\
\hline
\end{tabular}

\section{Discussion}

To the best of our knowledge, this is the first investigation that explored anxiety, depression, burden, quality of life and coping strategies used by informal primary caregivers of community-dwelling individuals with dementia in Malta. Determining the psychological wellbeing and type of coping strategies adopted by this category of caregivers could aid in developing community services that address their needs. This is in agreement with the Call for Action adopted by the First WHO Ministerial Conference on Global Action Against Dementia that emphasised that policy interventions should be sensitive to the specific needs of people living with dementia and their caregivers (17).

The findings reported here showed that more than half of participating caregivers experienced anxiety and with a quarter feeling depressed. Similar to other findings (18, $19)$, caregivers having higher levels of education scored significantly lower in anxiety and depression possibly indicating that education might act as a protective effect. Of note was the absence of depression in the twothirds of informal primary caregivers. People caring for individuals with dementia respond to depression in different ways and at different times. They might experience emotional distress soon after diagnosis of dementia in their relative, whereas in others as dementia progresses to its severe stage and the duration of care increases (20). However, the latter was not supported by the present findings in which the levels of anxiety, depression, burden and quality of life in informal primary caregivers were not found to be related to the number of years and number of hours per day spent in caregiving. Even though most caregivers participating in this study appeared to experience lower levels of depression, more research is needed to determine how the changing course of dementia might impact the caregiver emotional response in the local context.

Factors that strongly impacted caregiver burden in 
this study were the younger age of the individual with dementia, loss of functional status and a formal diagnosis of anxiety and depression in the caregiver. Although caregivers taking care of an individual with dementia with greater functional dependency experienced higher burden levels, no association between burden and patient cognitive scores was found possibly suggesting that deterioration in individuals with dementia abilities to take care of themselves leads to higher caregiver burden compared to cognitive deterioration. Similar trends were observed in other countries in which the direct influence of patients' cognition on caregiver burden is limited with the patients' functional abilities being the main predictor for burden (21). Contrasting previous data (22, 23), the age of the caregiver was not found to be related to burden. Rather, the age of the care-recipient showed a significant effect, with a younger age associated to higher caregiver burden. This is not surprising considering that young individuals with dementia pose additional challenges on their primary caregivers such as lack of appropriate access to specialized care as well as work and additional family commitments (24).

In caregivers, dysfunctional coping strategies were found to be significantly related to anxiety and depression, the duration of caregiving and the age of the care-recipient. Emotion-focus coping strategies, with coping styles that included acceptance and positive reframing, were preferred by the majority of caregivers and this may have contributed to a reduction in the anxiety and depression scores. Conversely, dysfunctional coping was mostly related to an increase in caregiver burden. Of note was that positive reframing coping style, in which stressful events are redefined in order to make them manageable (25), showed a significant correlation with emotional distress status, quality of life and caregiver burden. The ability to use this coping style in the present study was possibly related to the cohort cultural context, in which dementia care is a family affair and a shared care arrangement between family members as a form of respite is the norm (2).

The current study has a number of limitations. The WHOQOL-BREF instrument was not specifically developed for caregivers of individuals with dementia, although there are precedents for its use in this population (26). The study made use of individuals with dementia and their informal primary caregivers attending a day hospital which may not be representative of the dementia caregiver population in Malta. Sample selection was not randomised and thus the possibility of selection bias cannot be excluded. The modest sample size may have also limited statistical power.

In conclusion, the results reported here indicate that informal primary caregivers of individuals with dementia living in the community are more likely to suffer from emotional distress, experience burden and have a lower quality of life. However, those caregivers who use emotion-focused strategies were found to be protected against emotional distress. This continues to highlight the need of identifying multi-component interventions that support informal caregivers in maintaining a lifestyle that improves their quality of life. Knowing that in Malta dementia care in the community mostly follows a shared familial arrangement, caregiver support should be tailored to offer caring styles that adjust according to the caregiver circumstances and needs. Furthermore, assessing the psychological wellbeing of informal caregivers of individuals with dementia together with providing the necessary information on coping strategies that would support their caregiving role should form part of the dementia-management process. The present research continues to add to the recommendations of the National Strategy for Dementia in the Malta in which the provision of holistic support services to communitydwelling individuals with dementia and their informal caregivers is one of the main priorities to be addressed (27).

Acknowledgements: The authors would like to thank the caregivers and individuals with dementia who participated in the study.

Conflict of interest: none

Funding: none

Ethical standards: Ethical approval for the study was granted by the Faculty of Social Wellbeing Ethics Committee and the Research Ethics Committee of the University of Malta.

\section{References}

1. Scerri A, Scerri C. Dementia in Malta: New prevalence estimates and projected trends. Malta Med J 2012;24:21-24.

2. Innes A, Abela S, Scerri C. The organisation of dementia care by families in Malta: The experiences of family caregivers. Dementia 2011;10:165-184.

3. Ettema TP, Dröes RM, de Lange J, Ooms ME, Mellenbergh GJ, Ribbe MW The concept of quality of life in dementia in the different stages of the disease. Int Psychogeriatr 2005;17:353-370.

4. Takai M, Takahashi M, Iwamitsu Y, Oishi S, Miyaoka H. Subjective experiences of family caregivers of patients with dementia as predictive factors of quality of life. Psychogeriatrics 2011;11:98-104.

5. Campbell P, Oyebode J, Job D et al. Determinants of burden in those who care for someone with dementia. Int J Geriatr Psychiatry 2008;23:1078-1085.

6. Li R, Cooper C, Bradley J, Shulman A, Livingston G. Coping strategies and psychological morbidity in family carers of people with dementia: a systematic review and meta-analysis. J Affect Disord 2012;139:1-11.

7. Cooper C, Katona C, Orrell M, Livingston G. Coping strategies, anxiety and depression in caregivers of people with Alzheimer's disease. Int J Geriatr Psychiatry 2008;23:929-936.

8. Kneebone II, Martin PR. Coping and caregivers of people with dementia. Br J Health Psychol 2003;8:1-17.

9. Afram B, Stephan A, Verbeek $\mathrm{H}$ et al. Reasons for institutionalization of people with dementia: informal caregiver reports from 8 European countries. J Am Med Dir Assoc 2014;15:108-116.

10. Folstein MF, Folstein SE, McHugh PR. "Mini-Mental State". A practical method for grading the cognitive state of patients for the clinician. J Psychiatr Res 1975;12:189-198.

11. Collin C, Wade DT, Davies S, Horne V. The Barthel ADL index: a reliability study. Int Disabil Stud 1988;10:61- 63.

12. Zigmond AS, Snaith RP. The hospital anxiety and depression scale. Acta Psychiatr Scand 1983;67:361-370.

13. World Health Organization. WHOQOL-BREF: introduction, administration, scoring and generic version of the assessment - field trial version. 1996. WHO, Geneva.

14. Zarit SH, Reever KE, Bach-Peterson J. Relatives of the impaired elderly: correlates of feelings of burden. Gerontologist 1980;20:649-655.

15. Carver CS. You want to measure coping but your protocol's too long: Consider the Brief COPE. Int J Behav Med 1997;4:92-100.

16. Cooper C, Katona C, Orrell M, Livingston G. Coping strategies and anxiety in caregivers of people with Alzheimer's disease: The LASER-AD study. J Affect Disord 2006;90:15-20.

17. World Health Organization. Call for Action by the participants in the First 
WHO Ministerial Conference on Global Action Against Dementia (Geneva, 16-17 March 2015). http://www.who.int/mental_health/neurology/ dementia/call_for_action_en.pdf?ua=1. Accessed 17 May 2018.

18. Bjelland I, Krokstad S, Mykletun A, Dahl AA, Tell GS, Tambs K. Does a higher educational level protect against anxiety and depression? The HUNT study. Soc Sci Med 2008;66:1334-1345.

19. Sansoni J, Vellone E, Piras G. Anxiety and depression in communitydwelling, Italian Alzheimer's disease caregivers. Int J Nurs Pract 2004;10:93100 .

20. Covinsky K, Newcomer R, Fox P et al. Patient and caregiver characteristics associated with depression in caregivers of patients with dementia. J Gen Intern Med 2003;18:1006-1014.

21. Bergvall N, Brinck, P, Eek D et al. Relative importance of patient disease indicators on informal care and caregiver burden in Alzheimer's disease. Int Psychogeriatr 2011;23:73-85.

22. Marvardi M, Mattioli P, Spazzafumo L et al. The Caregiver Burden Inventory in evaluating the burden of caregivers of elderly demented patients: results from a multicenter study. Aging Clin Exp Res 2005;17:46-53.
23. Serrano-Aguilar PG, Lopez-Bastida J, Yanes-Lopez V. Impact on healthrelated quality of life and perceived burden of informal caregivers of individuals with Alzheimer's disease. Neuroepidemiology 2006;27:136-142.

24. Millenaar JK, de Vugt ME, Bakker C et al. The Impact of Young Onse Dementia on Informal Caregivers Compared with Late Onset Dementia: Results from the NeedYD Study. Am J Geriatr Psychiatry 2016;24:467-474.

25. Kliewer W, Fearnow MD, Miller PA. Coping socialization in middle childhood: tests of maternal and paternal influences. Child Dev 1996;67:23392357.

26. Wang J, Xiao LD, He GP, Ullah S, De Bellis A. Factors contributing to caregiver burden in dementia in a country without formal caregiver support. Aging Ment Health 2014;18:986-996.

27. Scerri C. Empowering change: a national strategy for dementia in the Maltese Islands. 2015. Parliamentary Secretariat for the Rights of Persons with Disability and Active Ageing, Valletta, Malta. 